\title{
A method for optimization of time intervals between rolling cuts on sorting humps
}

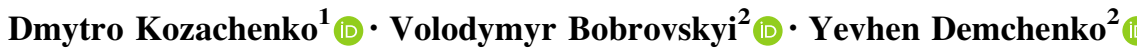

Received: 4 January 2017/Revised: 11 April 2018/Accepted: 16 April 2018/Published online: 11 May 2018

(C) The Author(s) 2018

\begin{abstract}
Sorting humps are the main technical means providing the breaking- and making-up of freight trains. Automation of sorting process by implementing microprocessor control systems is the main direction of increasing hump yard capacity, enhancement of safety in trains breaking-up, improvement of working conditions on hump yards. However, for wider introduction of such systems at the humps, it is necessary to reduce their cost. One of the main tasks of hump control systems is the determination of the optimal cars exit speeds from the retarders. Its solution is significantly complicated because of the uncertainty of cars rolling resistance coefficient, wind velocity and direction, retarder control errors. The paper is aimed at solving the problem of searching for such cars exit speeds from the retarders that provide the longest time intervals between them at the switches. Implementation of this method will make it possible to improve the quality of sorting process as a whole by improving the software of microprocessor control systems for automatic humping, rather than by increasing their technical facilities, which as a result will reduce the cost of their construction and operation. The research results, when solving the problem of searching for cars exit speeds from the retarders, allow us to move from searching for the maximum of a nonlinear non-smooth function of two variables to
\end{abstract}

Dmytro Kozachenko

kdntelefon@gmail.com

1 Research and Development Department, Dnipropetrovsk National University of Railway Transport Named After Academician V. Lazaryan, Dnipro 49010, Ukraine

2 Stations and Junctions Department, Dnipropetrovsk National University of Railway Transport Named After Academician V. Lazaryan, Dnipro 49010, Ukraine solution of an equation with one variable, which significantly accelerates and simplifies the solution to the problem.

Keywords Railways · Sorting hump · Optimization · Marshalling yard

\section{Introduction}

Sorting humps are the main technical means providing the breaking- and making-up of freight trains. Efficiency of their operation impacts largely on both the cost price and the safety of freight traffic on the railways. Automation of sorting process by implementing microprocessor control systems is the main direction of increasing hump yard capacity, enhancing safety in trains breaking-up, and improving working conditions on hump yards. However, the high complexity and cost of such systems limits their use on the railways. Therefore, the search for ways to improve microprocessor control systems for sorting humps, aimed at reducing their cost, is a crucial task.

When breaking up the cars of each train should be directed to the classification track in accordance with their purpose for the subsequent making-up of new trains. If in the process of rolling down of cars to different tracks of classification yard, the time intervals between them are not long enough for switching of points, some of the cars will be misrouted. As a result, there is a need to re-hump the cars, which leads to economic losses associated with additional operation of shunting locomotives and increase in the idle time of cars at the yard. In addition, accidental change of destination track of cars can lead to their overspeed coupling with the cars standing at the track, as a result, damaging the cars and cargoes. Therefore, providing 
time intervals between the cars necessary to prepare the routes for their rolling down to the desired destinations is an important task. Solution of this task will make it possible to reduce the cost of breaking- and making-up of trains at the yards and improve the safety of the humping process.

Various expensive automation systems for the sorting process, such as PROYARD III, Star II, MSR-32, and DDC III, have been created to improve the control of the process of breaking and making up the trains at the humps [1-4]. These systems regulate the process of car rolling from the hump, and for this purpose, determine the necessary exit speed from each retarder positions. To solve this problem, a significant number of various sensors are included in the automation system. They make it possible to specify the weight of cars, their running resistance, velocity and direction of wind, and other parameters necessary to control the retarders. Problems in controlling the process of cut rolling from the hump arise because of the errors in measuring these parameters, as well as because of random errors in controlling of cars, in the retarders, to the desired exit speed. We do not mean to lessen the importance of this approach, but it should be noted that there is an alternative approach for solving the problem. It is based on the improvement of the control algorithm of retarder positions and makes it possible to ensure a reliable separation of cuts at the switches even in the conditions of uncertainty.

The controlled objects at the humps are rolling single cars and groups of coupled cars that are called cuts. Contemporary algorithms for controlling the cars rolling speed at the humps are based on the differential equation of motion in which the independent variable is the distance:

$\mathrm{d} s=\frac{v \mathrm{~d} v}{g^{\prime}\left(i(s)-w_{\mathrm{rr}}-w_{\mathrm{pr}}(s, v)-w_{\mathrm{cr}}(s, v)-w_{\mathrm{ew}}(v)-b_{\mathrm{r}}(s)\right)}$,

where $g^{\prime}$ is the acceleration of gravity with wheels rotational inertia effect $\left(\mathrm{m} / \mathrm{s}^{2}\right) ; s$ is the distance from top of the hump to the first axle of the rolling cut (m); $v$ is cut velocity $(\mathrm{m} / \mathrm{s}) ; i(s)$ is variable gradient under the cut of cars $(\%) ; w_{\text {rr }}$ is the rolling resistance coefficient $(\mathrm{N} / \mathrm{kN}) ; w_{\mathrm{pr}}$ is the point resistance coefficient $(\mathrm{N} / \mathrm{kN}) ; w_{\mathrm{cr}}$ is curve resistance coefficient $(\mathrm{N} / \mathrm{kN}) ; w_{\mathrm{ew}}$ is environment and wind resistance coefficient $(\mathrm{N} / \mathrm{kN})$; and $b_{\mathrm{r}}$ is retarders resistance coefficient $(\mathrm{N} / \mathrm{kN})$

The complexity of the task of controlling breaking-up of train at the humps is connected with the fact that the values of the parameters $w_{\mathrm{rr}}, w_{\mathrm{pr}}, w_{\mathrm{cr}}$, and $w_{\mathrm{ew}}$ necessary for its solution are not exactly known. Moreover, since the braking positions realize the prescribed exit speeds for cut with errors, then the value of $b_{\mathrm{r}}$ in expression (1) is also not precisely known.
There is a considerable amount of scientific papers $[5,6]$ aimed at the development of technical means and methods for determining the coefficients of resistance to motion for expression (1) during the rolling-down process and improving the quality of implementation by retarders of the predetermined speeds of cut exit from the braking positions [7]. However, solving the problem solely by means of this approach leads, firstly, to increased cost of the train breaking-up control systems; secondly, it does not allow to completely exclude the action of random factors, as the resistance to car motion varies during the rolling process.

It should be noted that the possibility of an operative reaction to the deviation of the actual time of car movement from the calculated one exists in the systems using the semi-continuous car speed control [8]; however, due to a number of technical constraints, such systems are not used to regulate time intervals between cars at sloping part of large humps.

The problem of car speed control at humps was considered by Bessonenko et al. [9]. They proposed to set the cut exit speed from the first braking position, proceeding from the conditions of preserving the initial interval between the cuts at the hump top and ensuring their separation at the second brake position, and the braking mode at the second position-from the condition of cut separation at the switches. Some other researchers $[10,11]$ proposed to solve the problem of controlling the car rolling speed at the humps based on neural network training. The problem with this approach is that the methods based on the use of neural networks are heuristic and often lead to ambiguous solutions; as a result, the behavior of the trained neural network cannot always be clearly predicted. This increases the risk of their use in real-time systems related to traffic safety.

Thus, currently, the task of searching for optimal braking modes of cuts at sorting humps is yet to be performed and requires further research.

This paper is aimed at solving the problem of searching for such controlling actions of retarders on rolling cars, which allow creating the longest time intervals between them at the switches. This makes it possible to guarantee the sufficient time for switch operation between rolling cars when random changes in the braking modes occur. Implementation of this method will make it possible to improve the quality of sorting process as a whole by improving the software of automated control systems for automatic humping, rather than by increasing their technical facilities, which as a result will reduce the cost of their construction and operation.

The remainder of this paper is organized as follows: The statement of the problem of optimization of time intervals between rolling cuts in the design group is formulated in Sect. 2. This section also presents the dependence of 


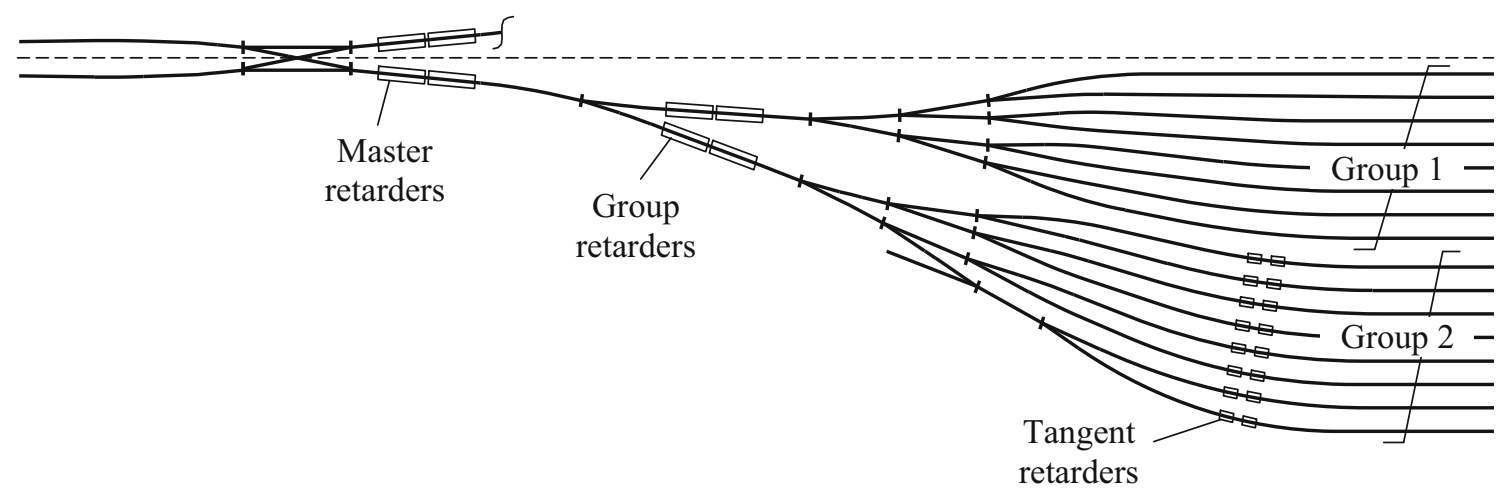

Fig. 1 Schematic view of the horizontal layout of sorting hump

intervals at separating elements on the braking mode of middle cut. The developed algorithm for optimization of the intervals between rolling cars on the sorting humps is given in Sect. 3. Finally, the conclusion is presented in Sect. 4.

\section{Optimization of time intervals between rolling cuts in the design group}

\subsection{Statement of the problem}

The research was carried out for a sorting hump equipped with two positions of clasp retarders at sloping part. The classification tracks in this research can be equipped with clasp as well as DOWTY retarders. This type of humps is the most prevalent at the marshalling yards. A schematic view of the horizontal layout of such sorting hump is given in Fig. 1.

For such conditions, the set of required exit speeds of the $i$ th cut from the brake positions at the hump slope can be presented by the vector $\boldsymbol{v}_{i}=\left\{v_{\mathrm{MRP}, i}, v_{\mathrm{GRP}, i}\right\}$, where $v_{\mathrm{MRP}, i}, v_{\mathrm{GRP}, i}$ are required exit speeds for master retarder (MRP) and group retarder (GRP). In this regard, the vector $\boldsymbol{v}_{i}=\left\{v_{\mathrm{MRP}, i}, v_{\mathrm{GRP}, i}\right\}$ will be referred to as braking modes of the cut. Braking modes of the cut $\boldsymbol{v}_{i}$ are restricted. These restrictions are applied by the design parameters of the hump, retarding mechanisms capacity of retarder positions, the requirements of the target regulation of cut speed on tangent retarder position (TRP), and the permissible speed of their entry to the car retarder and others. The set of permissible braking modes can be represented as a closed domain $\Omega_{\mathrm{d}}$. An example of such domain is shown in Fig. 2, and the principles of its construction are given in [12].

The domain of permissible braking modes in Fig. 2 has the following restrictions: 1 -on the highest possible speed of the cut entry to GRP; 2 - on the minimum speed of cut entry to the TRP; 3 -on TRP capacity; 4 -on the speed of

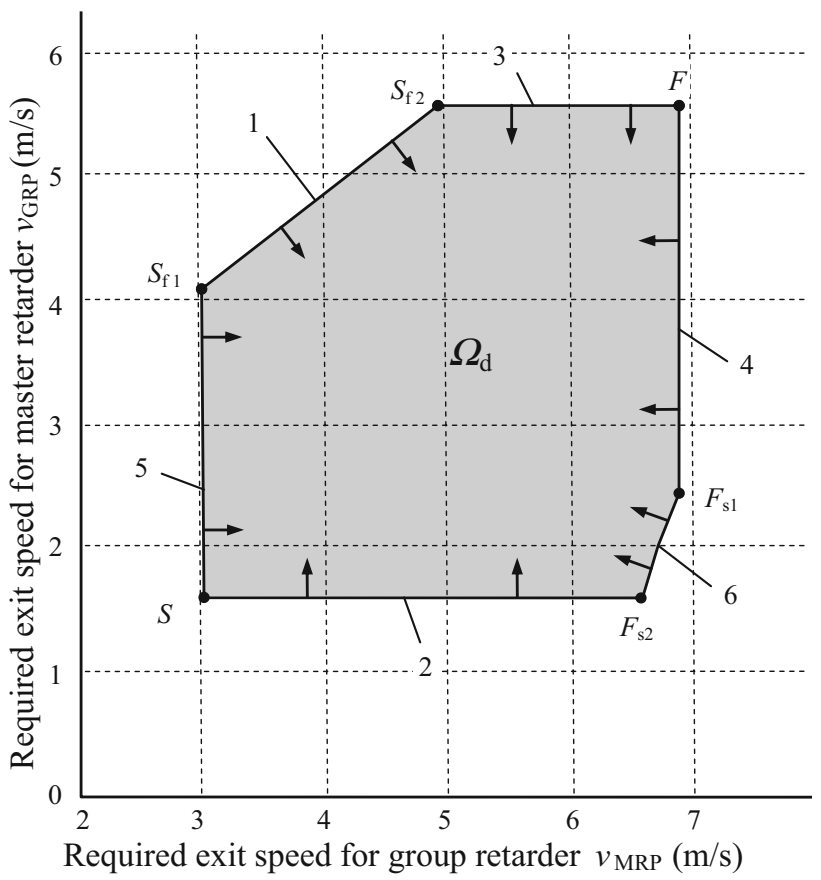

Fig. 2 Domain of permissible braking modes of cut

cut entry to the GRP; 5-on MRP capacity; 6-on GRP capacity. The intersection points of the restrictions correspond to the vertices of the domain $\Omega_{\mathrm{d}}: F, S, S_{\mathrm{f} 1}, S_{\mathrm{f} 2}, F_{\mathrm{s} 1}$, and $F_{\mathrm{s} 2}$.

It is evident that the braking mode of the $i$ th cut influences the intervals $\delta t$ with the preceding and subsequent cuts. The conducted research [13] showed that the problem of selecting the braking mode for all cuts of cars in the train can be solved on the basis of multiple local optimizations of braking modes in design groups of three adjacent cuts of this train. It is necessary to analyze the influence of braking mode of the $i$ th cut $\boldsymbol{v}_{i}$ on the intervals $\delta t_{i-1}$ and $\delta t_{i}$ at certain fixed braking modes $\boldsymbol{v}_{i-1}$ and $\boldsymbol{v}_{i+1}$ of the adjacent cuts. The value of these intervals is determined as 
$\delta t_{i-1}\left(\boldsymbol{v}_{i}\right)=t_{0, i-1}+t_{i}\left(\boldsymbol{v}_{i}, \sigma_{i-1}\right)-\tau_{i-1}\left(\boldsymbol{v}_{i-1}, \sigma_{i-1}\right)$,

$\delta t_{i}\left(\boldsymbol{v}_{i}\right)=t_{0, i}+t_{i+1}\left(\boldsymbol{v}_{i+1}, \sigma_{i}\right)-\tau_{i}\left(\boldsymbol{v}_{i}, \sigma_{i}\right)$,

where $t_{0, i-1}$ and $t_{0, i}$ are the initial intervals at the crest of hump between the $(i-1)$ th and $i$ th cuts and the $i$ th and $(i+1)$ th cuts, respectively; $t(\boldsymbol{v}, \sigma)$ is the cut rolling time from the moment of separation until the moment of occupation of the insulated section (IS) of separating point $\sigma$ at the installed braking mode $\boldsymbol{v}$; and $\tau(\boldsymbol{v}, \sigma)$ is the cut rolling time from the moment of separation until the moment of IS release of separating point $\sigma$.

It should be noted that, for a design group of three cuts with the controlled middle cut, the values $\tau_{1}$ and $t_{3}$ are constant, as the braking modes of the first and third cuts of the group do not vary; the initial intervals $t_{0}$ are also constant since the breaking-up speed in the framework of this task is taken as a constant.

The main method of hump processes research is currently the method of mathematical simulation of cuts rolling, for the implementation of which the motion differential equations of cuts of different forms are used. The principles of modeling are presented in Refs. $[14,15]$. In this paper, the computer simulation of cuts rolling from hump is conducted to evaluate the speed and time of their rolling. The principles of the model construction are presented in [16].

Taking into account the fact that the resistance coefficients of cars and the actual speeds of cuts exit of retarder positions are random variables, then the values of cuts movement time along the route $t(\boldsymbol{v}, \sigma)$, denoted by $\tau(\boldsymbol{v}, \sigma)$, as well as the intervals $\delta t$ in expressions (2) and (3) may differ from the calculated ones.

Under these circumstances, it is advisable to carry out the choice of optimal braking modes of cuts using the Wald criterion [17]. Using this criterion for determining the best braking mode for the middle cut in the design group of three cuts provides a maximum increase in the smaller of the two intervals in the group:

$\min \left\{\delta t_{1}\left(\boldsymbol{v}_{2}\right), \delta t_{2}\left(\boldsymbol{v}_{2}\right)\right\} \rightarrow \max$.

In this case, the possible time reserves at separating elements $\min \left\{\delta t_{1}\left(\boldsymbol{v}_{2}\right), \delta t_{2}\left(\boldsymbol{v}_{2}\right)\right\}-t_{\min }$ are used to ensure the cuts separation when the actual time of their rolling along the route is different from the calculated one (here, $t_{\min }$ is the minimum permissible value of the interval between the cuts, at which the point can be switched).

It should be noted there are possibly some cases of the particular location of separating points and retarder positions when one or both retarder positions do not influence the value of one or both separating intervals. The solution of the task of searching for optimal braking mode in such cases is trivial, and it is not considered in this paper.

\subsection{Dependence of intervals at separating elements on the braking mode of middle cut}

Figure 3 shows a graphic interpretation of the task of searching for the optimal braking mode of the middle cut in a design group. At this, each braking mode can be associated with the point in space, the abscissa $v_{\mathrm{MRP}, i}$ and ordinate $v_{\mathrm{GRP}, i}$, which present the cut exit speeds from the master and group retarder positions, respectively, and the applicate is the interval $\delta t$ with adjacent cut at the separating element. Scalar fields of the specified points $\left(v_{\mathrm{MRP}, 2}, v_{\mathrm{GRP}, 2}, \delta t_{1}\right)$ and $\left(v_{\mathrm{MRP}, 2}, v_{\mathrm{GRP}, 2}, \delta t_{2}\right)$ form two surfaces in the space, $\Theta_{1}$ and $\Theta_{2}$, respectively.

In general, the problem of determining the optimal braking mode at three-position sorting hump represents the task of searching for a maximum of nonlinear, non-smooth function of two variables formed by combining the surfaces $\Theta_{1}$ and $\Theta_{2}$. Determination of the maximum of this function can be performed by classical methods of direct search; however, their use is associated with significant time expenditures. In this regard, to solve the real-time problems, it is necessary to accelerate the search for an optimal solution, which can be achieved by studying the properties of expressions (2) and (3).

Further studies are based on the joint analysis of the domain of permissible braking modes $\Omega_{\mathrm{d}}$ and projection of the line $W\left(\boldsymbol{v}_{2}\right)$, which is formed by crossing of the fields of points $\Theta_{1}$ and $\Theta_{2}$ onto the plane $v_{\mathrm{MRP}, 2} O v_{\mathrm{GRP}, 2}$. Taking

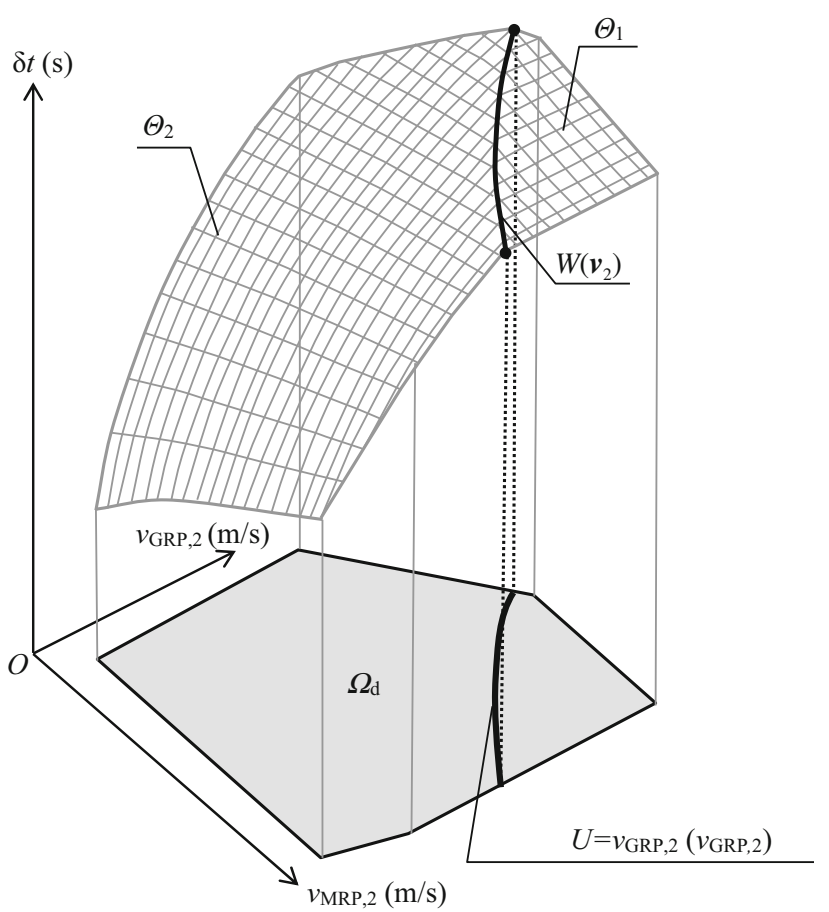

Fig. 3 Graphical interpretation of the task of searching for the optimal braking mode of middle cut in design group of three cuts 


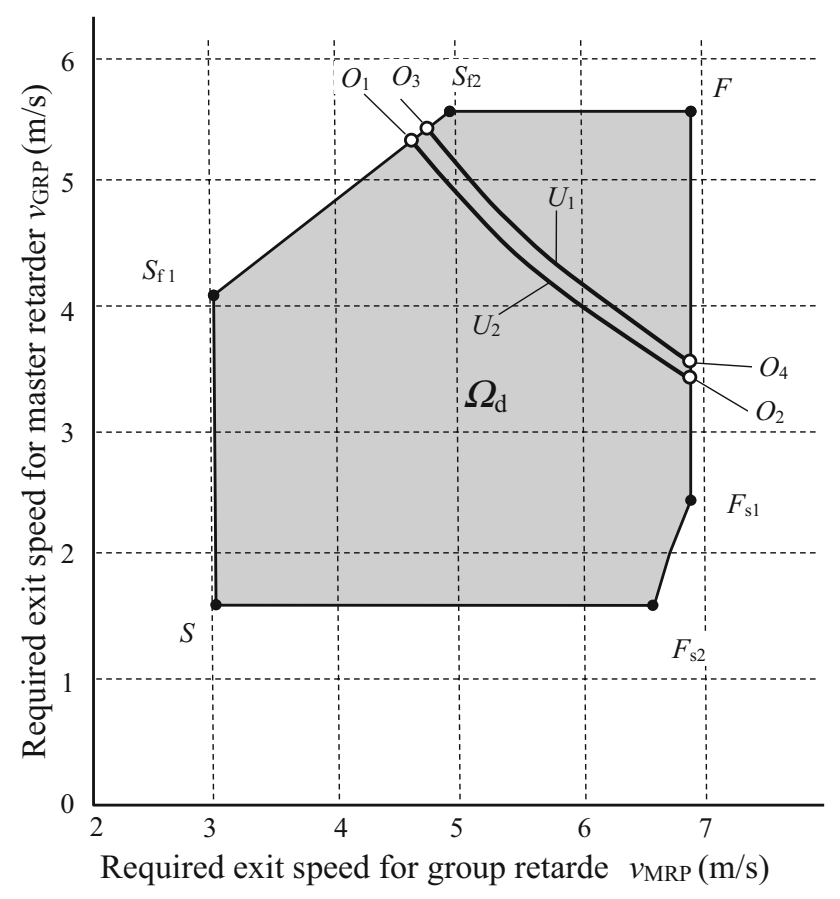

Fig. 4 Lines of equal intervals $U_{1}$ and $U_{2}$ in the domain of permissible braking modes $\Omega_{\mathrm{d}}$

into account that $\delta t_{1}=\delta t_{2}$ on the line $W\left(\boldsymbol{v}_{2}\right)$, then projection of the line $W$ onto plane $v_{\mathrm{MRP}, 2} O v_{\mathrm{GRP}, 2}$ will be called "line of equal intervals." An example of such projections is presented in Fig. 4. In this example, the line $U_{1}$ corresponds to the case when separation of cuts in the first pair takes place at the point $\sigma_{1}=3$, and in the second one at the point $\sigma_{2}=5$; the line $U_{2}$ corresponds to the case when the cuts separation in the first pair takes place at the point $\sigma_{1}=5$, and in the second one at the point $\sigma_{2}=3$. In this case, the line $U_{1}$ intersects the boundary of the domain $\Omega_{\mathrm{d}}$ at the points $O_{1}$ and $O_{2}$, and the line $U_{2}$ at the points $O_{3}$ and $O_{4}$.

Let us firstly consider the cases when surfaces $\Theta_{1}$ and $\Theta_{2}$ are not crossed within the domain $\Omega_{\mathrm{d}}$. If for all modes $\boldsymbol{v}_{2} \in \Omega_{\mathrm{d}}$, the inequality $\delta t_{1}\left(\boldsymbol{v}_{2}\right)<\delta t_{2}\left(\boldsymbol{v}_{2}\right)$ is valid, then expression (4) takes the form $\delta t_{1}\left(\boldsymbol{v}_{2}\right) \rightarrow$ max, and taking into account expression (2), we get $t_{2}\left(\boldsymbol{v}_{2}, \sigma_{1}\right) \rightarrow \max$. In this case, the optimal value of expression (4) provides a braking mode in which the controlled cut has the maximal rolling time from hump crest to the IS of the first separating point. The vertex $S$ of the domain $\Omega_{\mathrm{d}}$ (see Fig. 4), in which the cut exit speeds from the master and group retarder positions are minimal (slow mode of rolling down), corresponds to such a mode.

If for all modes $\boldsymbol{v}_{2} \in \Omega_{\mathrm{d}}$, the inequality $\delta t_{1}\left(\boldsymbol{v}_{2}\right)>\delta t_{2}\left(\boldsymbol{v}_{2}\right)$ is valid, then expression (4) takes the form $\delta t_{2}\left(\boldsymbol{v}_{2}\right) \rightarrow \max$, and taking into account expression (3), we get $\tau_{2}\left(\boldsymbol{v}_{2}, \sigma_{2}\right) \rightarrow \min$. In this case, the optimal value of expression (4) provides a braking mode in which the controlled cut has the minimal rolling time from hump crest to the IS of the first separating point. The vertex $F$ of the domain $\Omega_{\mathrm{d}}$ (see Fig. 4), in which the exit speeds from the master and group retarder positions are maximal (fast mode of rolling down), corresponds to such mode.

Next, let us consider the case when the surfaces $\Theta_{1}$ and $\Theta_{2}$ are crossed within the domain $\Omega_{\mathrm{d}}$. Taking into account that increase of braking of the middle cut leads to increase of the interval $\delta t_{1}$ and, thus, to reduction of $\delta t_{2}$, then condition (4) is equivalent to the condition:

$\delta t_{1}\left(\boldsymbol{v}_{2}\right)=\delta t_{2}\left(\boldsymbol{v}_{2}\right)=\overline{\overline{\delta t}}\left(\boldsymbol{v}_{2}\right) \rightarrow \max$.

For this reason, if the surfaces $\Theta_{1}$ and $\Theta_{2}$ are crossed within $\Omega_{\mathrm{d}}$, the maximum value of the criterion (4) will always be at the line $W=\Theta_{1} \cup \Theta_{2}$ of intersection of the surfaces $\Theta_{1}$ and $\Theta_{2}$. At this, the optimal braking mode $\boldsymbol{v}_{2}$ will be located on the line of equal intervals $U$.

Based on expressions (2), (3), and (5), the value of separating intervals that correspond to braking modes of the line $U$ can be determined using the expression

$$
\begin{aligned}
\overline{\overline{\delta t}} & =\frac{\delta t_{1}+\delta t_{2}}{2} \\
& =\frac{t_{0,1}+t_{2}\left(\boldsymbol{v}_{2}, \sigma_{1}\right)-\tau_{1}\left(\sigma_{1}\right)+t_{0,2}+t_{3}\left(\sigma_{2}\right)-\tau_{2}\left(\boldsymbol{v}_{2}, \sigma_{2}\right)}{2} .
\end{aligned}
$$

Let us designate

$A=\frac{t_{0,1}-\tau_{1}\left(\sigma_{1}\right)+t_{0,2}+t_{3}\left(\sigma_{2}\right)}{2} ;$

then,

$\overline{\overline{\delta t}}=A+\frac{t_{2}\left(\boldsymbol{v}_{2}, \sigma_{1}\right)-\tau_{2}\left(\boldsymbol{v}_{2}, \sigma_{2}\right)}{2}$.

Considering the fact that at the fixed braking modes of the first and third cuts, the value of the summand $A$ is constant, then the value of separating intervals $\overline{\overline{\delta t}}$ will depend only on the diminution $t_{2}\left(\boldsymbol{v}_{2}, \sigma_{1}\right)-\tau_{2}\left(\boldsymbol{v}_{2}, \sigma_{2}\right)$. It is defined both by mutual arrangement of the points $\sigma_{1}$ and $\sigma_{2}$ on the hump plan and by braking mode $\boldsymbol{v}_{2}$ of the middle cut at MRP and GRP; wherein condition (4) can be written as

$t_{2}\left(\boldsymbol{v}_{2}, \sigma_{1}\right)-\tau_{2}\left(\boldsymbol{v}_{2}, \sigma_{2}\right) \rightarrow \max$

under the constraint that provides equality of intervals

$B\left(\boldsymbol{v}_{2}\right)=B\left(\boldsymbol{v}_{1}, \boldsymbol{v}_{3}\right)$,

where

$B\left(\boldsymbol{v}_{2}\right)=t_{2}\left(\boldsymbol{v}_{2}, \sigma_{1}\right)+\tau_{2}\left(\boldsymbol{v}_{2}, \sigma_{2}\right)$,

$B\left(\boldsymbol{v}_{1}, \boldsymbol{v}_{3}\right)=t_{0,2}-t_{0,1}+\tau_{1}\left(\sigma_{1}, \boldsymbol{v}_{1}\right)+t_{3}\left(\sigma_{2}, \boldsymbol{v}_{3}\right)$.

Graphically, expressions (6) and (7) are illustrated in Fig. 5. The value of separating intervals is determined by the difference of vectors of $C D$ and $E F$; to ensure their 
(a)

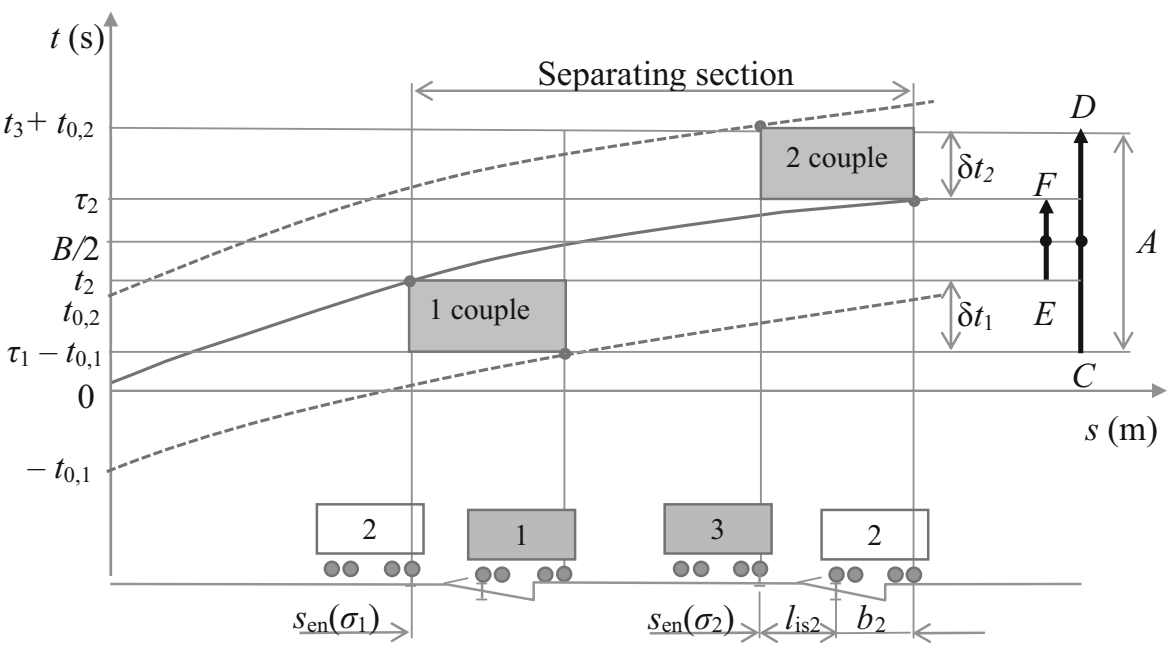

(b)

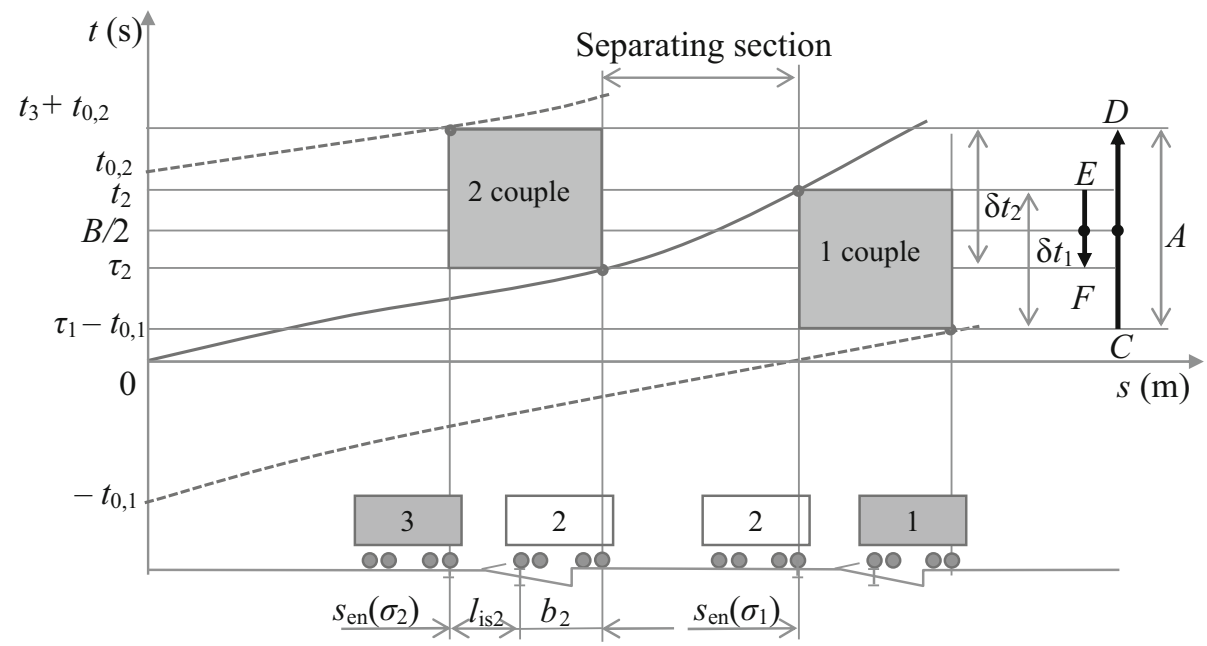

Fig. 5 Equality conditions of separating intervals at the point switches: $\mathbf{a} \sigma_{1}<\sigma_{2}$; b $\sigma_{1}>\sigma_{2}$

equality, the ordinate of the middle of the vector $E F$ must coincide with the ordinate of the middle of the vector $C D$.

Let us investigate the nature of change in the value of separating intervals $\delta t_{1}=\delta t_{2}$ along the line $U$ and introduce the concept of area of cuts separation in design group. This area is located between the points of middle cut entry to the separating point $\sigma_{1}$ and its exit from the separating point $\sigma_{2}$. The length of this area can be defined as

$l_{\mathrm{ss}}=\left|s_{\mathrm{en}}\left(\sigma_{1}\right)-s_{\mathrm{ex}}\left(\sigma_{2}\right)\right|$,

where $s_{\mathrm{en}}$ and $s_{\mathrm{ex}}$ are the coordinates of the entry and exit from the IS of the separating points, respectively.

Considering that the time $t(s)$ increases monotonically with an increase in rolling distance $s$, the sign and value of the mentioned diminution will depend on the correlation of coordinates of the point of middle cut entry $s_{\mathrm{en}}\left(\sigma_{1}\right)$ to IS of the first point $\sigma_{1}$ and the exit point of this cut from IS of the second point $\sigma_{2}$.

The coordinate $s_{\mathrm{en}}\left(\sigma_{1}\right)$ is determined by the distance from the top of a hump to the beginning of IS of the point $\sigma_{1}$ and is a constant. Moreover, the coordinate $s_{\mathrm{ex}}\left(\sigma_{2}\right)$ depends on the base length of the middle cut $b_{2}$ and it is also a constant:

$s_{\mathrm{ex}}\left(\sigma_{2}\right)=s_{\mathrm{en}}\left(\sigma_{2}\right)+l_{\mathrm{is} 2}+b_{2}$,

where $l_{\text {is } 2}$ is the length of insulated section of the point $\sigma_{2}$.

Let us designate $t_{2}\left(\sigma_{1}\right)=t\left(s_{\mathrm{en}}\left(\sigma_{1}\right)\right)$ and $\tau_{2}\left(\sigma_{2}\right)=t\left(s_{\mathrm{ex}}\left(\sigma_{2}\right)\right)$. In this case, if $s_{\mathrm{en}}\left(\sigma_{1}\right)<s_{\mathrm{ex}}\left(\sigma_{2}\right)$, then $t_{2}\left(\sigma_{1}\right)<\tau_{2}\left(\sigma_{2}\right)$; on the contrary, if $s_{\mathrm{en}}\left(\sigma_{1}\right)>s_{\mathrm{ex}}\left(\sigma_{2}\right)$, then $t_{2}\left(\sigma_{1}\right)>\tau_{2}\left(\sigma_{2}\right)$. Consider the various options for the mutual arrangement of separating points of the first and second pairs of cuts.

Assume that 


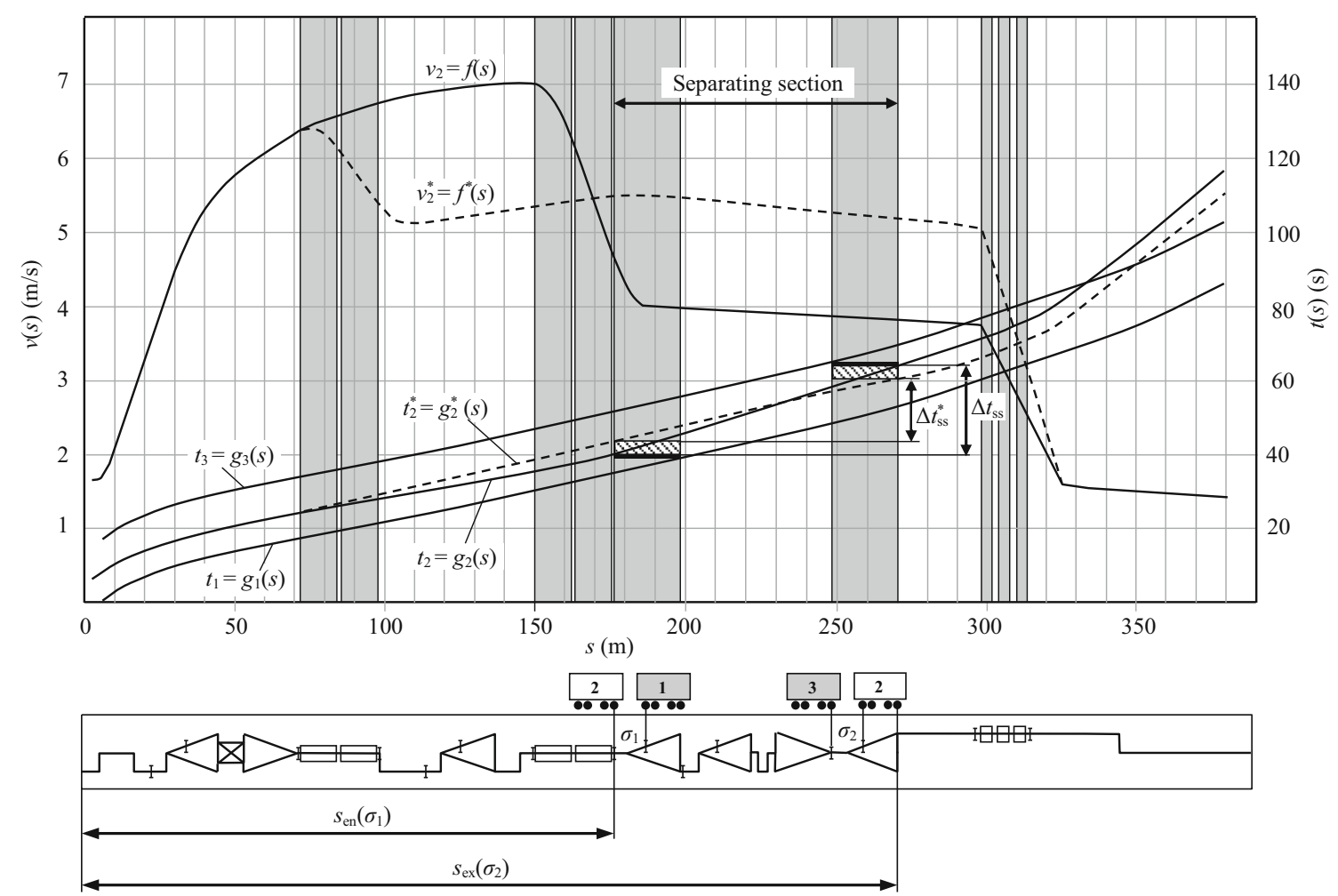

Fig. 6 Control scheme of separating intervals at the point switches with $s_{\mathrm{en}}\left(\sigma_{1}\right)<s_{\mathrm{ex}}\left(\sigma_{2}\right)$

$s_{\mathrm{en}}\left(\sigma_{1}\right)<s_{\mathrm{ex}}\left(\sigma_{2}\right)$

is fulfilled (see Fig. 5a). In this case, as shown above, the diminution $\Delta t_{\mathrm{ss}}$ is negative:

$\Delta t_{\mathrm{ss}}=t_{2}\left(\sigma_{1}\right)-\tau_{2}\left(\sigma_{2}\right)<0$.

Consequently, to simultaneously increase the intervals $\delta t_{1}$ and $\delta t_{2}$ and to keep their equality $\delta t_{1}=\delta t_{2}$, we should choose a braking modes $\boldsymbol{v}_{2}$ of the middle cut such that the value of diminution $\left|\Delta t_{\mathrm{ss}}\right|$, which can be regarded as the time of occupation of separating section by the middle cut of the group, would be minimal. The value $\left|\Delta t_{\mathrm{ss}}\right|$ is inversely proportional to the average speed of cut $\bar{v}_{\mathrm{ss}}$ at the section of separation of cuts $1-2$ and $2-3\left[s_{\mathrm{en}}\left(\sigma_{1}\right), s_{\mathrm{ex}}\left(\sigma_{2}\right)\right]$ :

$\left|\Delta t_{\mathrm{ss}}\right|=\frac{\left|s_{\mathrm{en}}\left(\sigma_{1}\right)-s_{\mathrm{ex}}\left(\sigma_{2}\right)\right|}{\bar{v}_{\mathrm{ss}}}$.

The control scheme of separating intervals at the point switches with $s_{\mathrm{en}}\left(\sigma_{1}\right)<s_{\mathrm{ex}}\left(\sigma_{2}\right)$ is shown in Fig. 6. This figure presents the following dependencies:

$t_{1}=g_{1}(s)$ and $t_{3}=g_{3}(s)$-dependencies of the rolling time for the first and third cuts from the coordinate of the first axis of the cut $s$ relative to the top of the hump, respectively;

$t_{2}=g_{2}(s)$ and $v_{2}=f_{2}(s)$-dependencies of the time and speed of rolling of the second cut from the coordinate $s$ at some initial braking mode, respectively; $t_{2}^{*}=g_{2}^{*}(s)$ and $v_{2}^{*}=f_{2}^{*}(s)$-dependencies of the time and speed of rolling of the second cut from the coordinate $s$ under the changed braking mode, respectively.

The dependencies $g_{1}(s), g_{2}(s), g_{2}^{*}(s), g_{3}(s), f_{2}(s)$, and $f_{2}^{*}(s)$ are obtained as a result of the simulation of the controlled rolling of the cuts from the sorting hump.

To increase the intervals $\delta t_{1}$ and $\delta t_{2}$, while maintaining their equality, the average speed of cut should be increased at the section $\left[s_{\mathrm{en}}\left(\sigma_{1}\right), s_{\mathrm{ex}}\left(\sigma_{2}\right)\right]$ and thereby the diminution $\left|\Delta t_{\mathrm{ss}}\right|$ should be reduced to the value $\left|\Delta t_{\mathrm{ss}}^{*}\right|$. At the same time, it is necessary to reduce the speed of cut at the initial section of rolling down from the top of the hump to the point $s_{\text {en }}\left(\sigma_{1}\right)$ to increase the time $t_{2}\left(\sigma_{1}\right)$. The timeline of cut rolling down at this mode is shown in Fig. 6 by the dotted line.

Such mode of motion can be achieved at the corresponding speed reduction of the cut exit from MRP with a simultaneous increase in the exit speed from GRP. As a result, the optimal braking mode which provides maximal intervals at the separating points will be located at the intersection of a line of equal intervals with the boundary section of the domain of permissible braking modes with its round from the point $F$ to the point $S$ in a counterclockwise direction. As an example, the line $U_{1}$ (see Fig. 4) can be considered. At $s_{\mathrm{en}}\left(\sigma_{1}\right)=176.27 \mathrm{~m}$, $s_{\mathrm{en}}\left(\sigma_{2}\right)=248.49 \mathrm{~m}, \quad l_{\mathrm{is}}=11.38 \mathrm{~m}, \quad$ and $b_{2}=10.5 \mathrm{~m}$, 


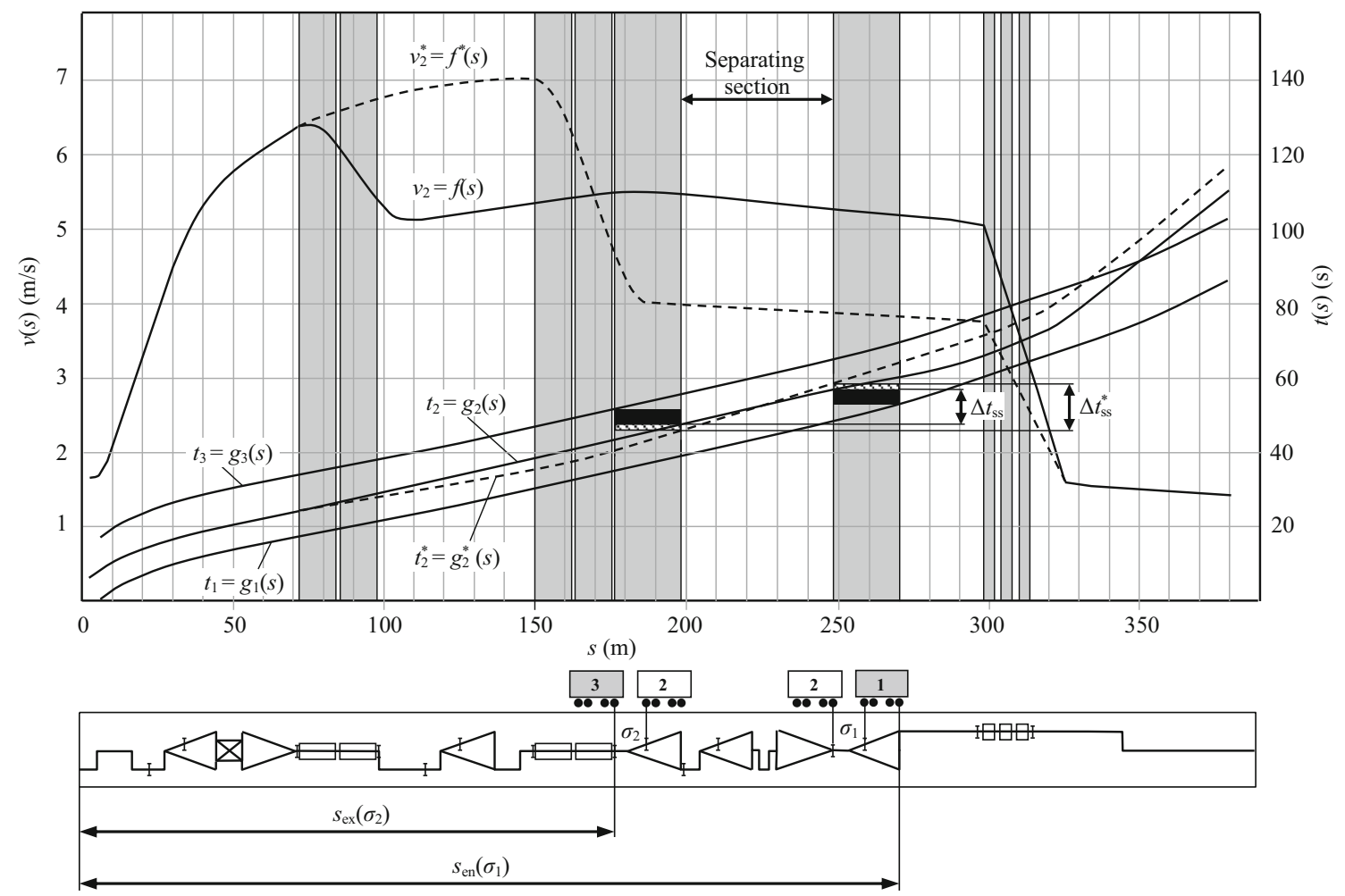

Fig. 7 Control scheme of separating intervals at the point switches with $s_{\mathrm{en}}\left(\sigma_{1}\right)>s_{\mathrm{ex}}\left(\sigma_{2}\right)$

condition (11) is fulfilled and the optimal braking mode corresponds to the point $O_{1}=\{4.69 ; 5.33\}$ of the intersection of the line $U_{1}$ with the segment $S_{\mathrm{f} 1} S_{\mathrm{f} 2}$.

Another option of cuts separation occurs in the case

$s_{\mathrm{en}}\left(\sigma_{1}\right)>s_{\mathrm{ex}}\left(\sigma_{2}\right)$,

wherein

$\Delta t_{\mathrm{ss}}=t_{2}\left(\sigma_{1}\right)-\tau_{2}\left(\sigma_{2}\right)>0$.

Consequently, in accordance with (6), to simultaneously increase the intervals $\delta t_{1}$ and $\delta t_{2}$ and to keep their equality $\delta t_{1}=\delta t_{2}$, one should select a braking mode $\boldsymbol{v}_{2}$ of the middle cut such that the absolute value of the diminution $\Delta t_{\mathrm{ss}}$ in (15) would be maximal. Obviously, in accordance with (13), the value $\Delta t_{\mathrm{ss}}$ will increase with a decrease in the average speed of cut at the section $\left[s_{\mathrm{ex}}\left(\sigma_{2}\right), s_{\mathrm{en}}\left(\sigma_{1}\right)\right]$ (see Fig. 7).

At this, to increase both intervals $\delta t_{1}$ and $\delta t_{2}$ and at the same time to maintain their equality, it is necessary to reduce the movement time of cut at the initial section $\tau_{2}\left(\sigma_{2}\right)$ with the simultaneous increase of $\Delta t_{\mathrm{ss}}$. Thus, upon the condition $s_{\mathrm{en}}\left(\sigma_{1}\right)>s_{\mathrm{ex}}\left(\sigma_{2}\right)$, one should increase the cut exit speeds from MRP while decreasing the cut exit speeds from GRP. As a result, the optimal braking mode which provides maximal intervals at the separating points will be located at the point of intersection of equal interval lines with the boundary section of the domain of permissible braking modes with its round from the point $F$ to the point $S$ in a clockwise direction. At the same location of point switches 3 and 5 as in the first case and the same base of the middle cut, condition (15) is fulfilled and the optimal braking mode corresponds to the point $O_{4}=\{6.90 ; 3.54\}$ of intersection of the line $U_{2}$ with the segment $F F_{\mathrm{s} 1}$.

The limiting case takes place when $s_{\mathrm{en}}\left(\sigma_{1}\right)=s_{\mathrm{ex}}\left(\sigma_{2}\right)$. In this case, all the modes of the line $U$ provide an equal value of the intervals at the separating points.

Our study shows that the specialization of retarder positions on the sloping part of hump to perform the interval and the interval target regulation of cuts rolling speed is inappropriate. Rational distribution of the value of extinguished energy of cut between retarder positions depends on the location of the separating points along the route of rolling down and on the cut base length. At this, both MRP and GRP are involved in providing the necessary separating intervals and permissible speed of cut entry to the park retarder position.

As it follows from (2) and (3), the value of separating intervals is influenced not only by breaking modes of the middle cut but also by braking modes of the adjacent cuts. An important special case is the change of braking modes of the first and last cuts, at which the position of the line $U$ and hence the optimal braking mode of the middle cut remain unchanged. 


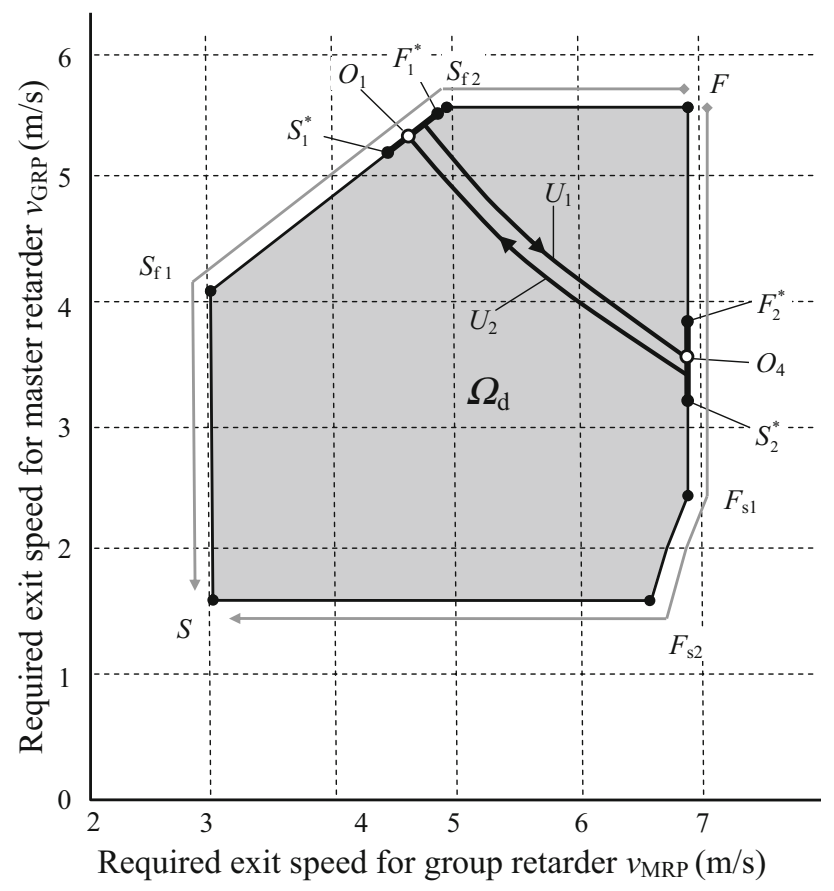

Fig. 8 Search for optimal modes in the domain of permissible braking modes

To investigate the influence of braking modes of the first and last cuts from the design group, expressions (2) and (3) will be considered. Let the braking mode of the first cut $\boldsymbol{v}_{1}$ be changed to some mode $v_{1}^{*}$, and the braking mode of the third cut $v_{3}$ to some mode $\boldsymbol{v}_{3}^{*}$. The change in regimes causes a change in the movement time of the first cut $\tau_{1}$ beyond separating point $\sigma_{1}$ for the value $\Delta_{1}$ and of the third cut $t_{3}$ before the separating point for the value $\Delta_{2}$, respectively:

$\tau_{1}\left(\boldsymbol{v}_{1}^{*}, \sigma_{1}\right)=\tau_{1}\left(\boldsymbol{v}_{1}, \sigma_{1}\right)+\Delta_{1}$,

$t_{3}\left(v_{3}^{*}, \sigma_{2}\right)=t_{3}\left(v_{3}, \sigma_{2}\right)+\Delta_{2}$.

Then, in accordance with (2) and (3), the intervals at separating points will be equal: $\delta t_{1}^{*}\left(\boldsymbol{v}_{2}\right)=t_{0,1}+t_{2}\left(\boldsymbol{v}_{2}, \sigma_{1}\right)-\tau_{1}\left(\boldsymbol{v}_{1}^{*}, \sigma_{1}\right)$,

$\delta t_{2}^{*}\left(\boldsymbol{v}_{2}\right)=t_{0,2}+t_{3}\left(\boldsymbol{v}_{3}^{*}, \sigma_{2}\right)-\tau_{2}\left(\boldsymbol{v}_{2}, \sigma_{2}\right)$.

Substituting expression (16) into (17) and (18), we obtain

$\delta t_{1}^{*}\left(\boldsymbol{v}_{2}\right)=t_{0,1}+t_{2}\left(\boldsymbol{v}_{2}, \sigma_{1}\right)-\tau_{1}\left(\boldsymbol{v}_{1}, \sigma_{1}\right)+\Delta_{1}$,

$\delta t_{2}^{*}\left(\boldsymbol{v}_{2}\right)=t_{0,2}+t_{3}\left(\boldsymbol{v}_{3}, \sigma_{2}\right)+\Delta_{2}-\tau_{2}\left(\boldsymbol{v}_{2}, \sigma_{2}\right)$

or

$\delta t_{1}^{*}\left(\boldsymbol{v}_{2}\right)=\delta t_{1}\left(\boldsymbol{v}_{2}\right)+\Delta_{1}$,

$\delta t_{2}^{*}\left(\boldsymbol{v}_{2}\right)=\delta t_{2}\left(\boldsymbol{v}_{2}\right)+\Delta_{2}$.

These expressions allow suggesting that if $\delta t_{1}\left(\boldsymbol{v}_{2}\right)=$ $\delta t_{2}\left(\boldsymbol{v}_{2}\right)$ and $\Delta_{1}=\Delta_{2}$, then $\delta t_{1}^{*}\left(\boldsymbol{v}_{2}\right)=\delta t_{2}^{*}\left(\boldsymbol{v}_{2}\right)$. Therefore, at $\Delta_{1}=\Delta_{2}$, the position of the $U$ line in the domain of permissible braking modes is unchanged. We take into account the fact that the direction of the interval value increases along the line $U$ and depends only on the position of separating points, and the length of the middle cut based on the position of optimal braking mode remains unchanged too. Thus, if it is known that braking mode $\boldsymbol{v}_{2}$ is the optimal one, the breaking modes change of the first and last cuts in such a way that $\Delta_{1}=\Delta_{2}$ and the optimal braking mode will remain at the same point $\boldsymbol{v}_{2}$.

Let some of the braking modes of the cuts from the design group $\boldsymbol{v}_{1}, \boldsymbol{v}_{2}$, and $\boldsymbol{v}_{3}$ provide equal separating intervals in the first and second pairs. When maintaining the braking mode of $\boldsymbol{v}_{2}$ and changing the braking mode of the first cut $v_{1}$ to the mode $v_{1}^{*}$ so that $\tau_{1}\left(v_{1}^{*}, \sigma_{1}\right)=\tau_{1}\left(\boldsymbol{v}_{1}, \sigma_{1}\right)+\Delta_{1}$, the intervals' equality in the first and second pairs will be kept only when with the changed braking mode of the third cut $v_{3}^{*}$, the condition $t_{3}\left(v_{3}^{*}, \sigma_{2}\right)=t_{3}\left(v_{3}, \sigma_{2}\right)+\Delta_{2}$ will be fulfilled. Taking into account the fact that when changing the braking modes of the first and third cuts which cause similar changes in the values $\tau_{1}\left(\boldsymbol{v}_{1}, \sigma_{1}\right)$ and $t_{3}\left(\boldsymbol{v}_{3}, \sigma_{2}\right)$, the position of $U$ line is not changed, it can be argued that only one line of equal intervals corresponds to each braking mode $\boldsymbol{v}_{2}$. As a

Table 1 Example of searching for the optimal braking mode in the group of three cuts with dividing points 3 and 5 in the first and second pairs

\begin{tabular}{|c|c|c|c|c|c|c|c|c|c|c|c|c|}
\hline \multirow[t]{3}{*}{ Step } & \multicolumn{6}{|l|}{ Beginning point } & \multicolumn{6}{|l|}{ Ending point } \\
\hline & \multirow[t]{2}{*}{ Name of point } & \multicolumn{2}{|l|}{ Mode } & \multirow[t]{2}{*}{$B\left(\boldsymbol{v}_{2}\right)$} & \multicolumn{2}{|c|}{ Intervals } & \multirow[t]{2}{*}{ Name of point } & \multicolumn{2}{|l|}{ Mode } & \multirow[t]{2}{*}{$B\left(\boldsymbol{v}_{2}\right)$} & \multicolumn{2}{|c|}{ Intervals } \\
\hline & & $v_{\mathrm{MRP}, 2}$ & $v_{\mathrm{GRP}, 2}$ & & $\delta t_{1}$ & $\delta t_{2}$ & & $v_{\mathrm{MRP}, 2}$ & $v_{\mathrm{GRP}, 2}$ & & $\delta t_{1}$ & $\delta t_{2}$ \\
\hline 1 & $F_{1}^{*}$ & 5.09 & 5.54 & 91.76 & 4.33 & 6.92 & $S_{1}^{*}$ & 4.48 & 5.19 & 96.57 & 6.12 & 4.23 \\
\hline 2 & $S_{\mathrm{f} 2}$ & 4.95 & 5.54 & 92.48 & 4.68 & 6.89 & $S_{1}^{*}$ & 4.48 & 5.19 & 96.57 & 6.12 & 4.23 \\
\hline 3 & $S_{\mathrm{f} 2}$ & 4.95 & 5.54 & 92.48 & 4.68 & 6.89 & - & 4.66 & 5.32 & 94.93 & 5.54 & 5.29 \\
\hline 4 & - & 4.77 & 5.41 & 93.32 & 4.55 & 5.92 & - & 4.66 & 5.32 & 94.93 & 5.54 & 5.29 \\
\hline$\vdots$ & $\vdots$ & $\vdots$ & $\vdots$ & $\vdots$ & $\vdots$ & $\vdots$ & $\vdots$ & $\vdots$ & $\vdots$ & $\vdots$ & $\vdots$ & $\vdots$ \\
\hline 12 & $O_{1}$ & 4.69 & 5.33 & 94.69 & 5.45 & 5.45 & $O_{1}$ & 4.69 & 5.33 & 94.69 & 5.45 & 5.45 \\
\hline
\end{tabular}


numerical characteristic of the equal interval line corresponding to the mode $\boldsymbol{v}_{2}$, the value of the parameter $B\left(\boldsymbol{v}_{2}\right)$ can be used. It is determined in accordance with expression (8). To analyze the correlation of the interval value in the first and second pairs of cuts when using the braking mode $\boldsymbol{v}_{2}$, after fixing the braking modes of the first and last cuts from group, it is sufficient to determine the value of the parameter $B\left(\boldsymbol{v}_{1}, \boldsymbol{v}_{3}\right)$ in accordance with expression (9) and compare it with the value of the parameter $B\left(v_{2}\right)$. If condition (7) is fulfilled, the intervals of both pairs are equal.

If the inequality

$B\left(\boldsymbol{v}_{2}\right)<B\left(\boldsymbol{v}_{1}, \boldsymbol{v}_{3}\right)$

takes place, the interval in the second pair of cuts is longer than that in the first pair, and the mode that will provide equal intervals is in the direction $S$ from this one. If the inequality

$B\left(v_{2}\right)>B\left(v_{1}, v_{3}\right)$

takes place, the interval in the second pair of cuts is shorter than that in the first pair, and the mode that will provide equal intervals is in the direction $F$ from the given one.

Expressions (21) and (22) allow us to formulate the conditions to check the positions of equal interval line with respect to two arbitrary braking modes $M$ and $N$ in the following way (let $B_{M}<B_{N}$ ):

- If the condition

$B_{M}<B\left(v_{1}, v_{3}\right)<B_{N}$

is fulfilled, the line of equal interval lies between the points corresponding to the modes $M$ and $N$.

- If $B\left(\boldsymbol{v}_{1}, \boldsymbol{v}_{3}\right)>B_{M}$ and $B\left(\boldsymbol{v}_{1}, \boldsymbol{v}_{3}\right)>B_{N}$, the line of equal intervals is shifted from the point corresponding to the mode $N$ in the direction coinciding with the direction of the vertex $F$ to the vertex $S$.

- If $B\left(\boldsymbol{v}_{1}, \boldsymbol{v}_{3}\right)<B_{M}$ and $B\left(\boldsymbol{v}_{1}, \boldsymbol{v}_{3}\right)<B_{N}$, the line of equal intervals is shifted from the point corresponding to the mode $M$, in the direction coinciding with the direction of the vertex $S$ to the vertex $F$.

It should be noted that braking modes of the first and last cuts of the calculated combination are also limited. The minimum possible value of the parameter $B\left(\boldsymbol{v}_{1}, \boldsymbol{v}_{3}\right)$ corresponds to the conditions when rolling down of the first and third cuts is performed in fast mode, and the maximum one when rolling down of these cuts is performed in slow mode. In these circumstances taking into account characteristic (7), optimal braking modes can only be in the section of border, for which the following condition is fulfilled:

$B_{F}^{*} \leq B\left(\boldsymbol{v}_{2}\right) \leq B_{S}^{*}$, where $\quad B_{F}^{*}=\max \left(B_{F}, B_{\min }\left(\boldsymbol{v}_{1}, \boldsymbol{v}_{3}\right)\right) ; \quad B_{S}^{*}=\min \left(B_{S}, B_{\max }\right.$ $\left.\left(\boldsymbol{v}_{1}, \boldsymbol{v}_{3}\right)\right) ; B_{\min }\left(\boldsymbol{v}_{1}, \boldsymbol{v}_{3}\right)$ and $B_{\max }\left(\boldsymbol{v}_{1}, \boldsymbol{v}_{3}\right)$ are the minimal and maximal value of the parameter $B\left(v_{1}, v_{3}\right)$ for the first and last cuts, respectively.

\section{Algorithm for optimization of time intervals between rolling cars on the sorting humps}

The performed studies make it possible to use the following algorithm for searching the optimal braking mode of the middle cut of cars in the design group.

At the preliminary stage, one should build the domain of the permissible braking modes $\Omega_{\mathrm{d}}$. For the nodal points of this domain, the parameters $B\left(\boldsymbol{v}_{2}\right)$ should be set. Based on the analysis of the coordinates $s_{\mathrm{en}}\left(\sigma_{1}\right)$ and $s_{\mathrm{ex}}\left(\sigma_{2}\right)$, it is necessary to find the section of the boundary at which the optimal braking mode of ( $S F$ or $F S$ ) is located and to identify the points $B_{F}^{*}$ and $B_{S}^{*}$ corresponding to the maximum possible braking modes of the first and last cuts from the design group. Further search is carried out in the following order:

1. After fixing the braking modes of the first and third cuts from design group, the value $B\left(\boldsymbol{v}_{1}, \boldsymbol{v}_{3}\right)$ should be set.

2. If $B\left(\boldsymbol{v}_{1}, \boldsymbol{v}_{3}\right) \leq B_{F}^{*}$, the optimal mode is the fast mode of rolling $F$. End of search.

3. If $B\left(\boldsymbol{v}_{1}, v_{3}\right) \geq B_{S}^{*}$, the optimal mode is the slow mode of rolling $S$. End of search.

4. On the selected section of the boundary, one should find the segment $M N$ for the vertices of which expression (23) is valid.

5. The optimal mode is determined at the segment $M N$ by searching for the root of the equation

$B(q)=B\left(\boldsymbol{v}_{1}, \boldsymbol{v}_{3}\right)$,

where $q$ is the linear parameter defining the position of the mode $\boldsymbol{v}_{2}$ at the segment $M N$.

For the example shown in Fig. 8, if separating points in the first and second pairs will be, respectively, the points 3 and 5 , the optimal braking modes will be located at the border section of the domain of permissible modes $F_{1}^{*} S_{1}^{*}$ where the parameter $B\left(v_{2}\right)$ is being changed in the range from 91.76 to 96.57 .

Let the rolling-down conditions of the design group cuts be $t_{0,1}=6.57, \quad t_{0,2}=9.81, \quad \tau_{1}\left(3, v_{1}\right)=39.43$, and $t_{3}\left(5, v_{3}\right)=52.02$; then, $B\left(\boldsymbol{v}_{2}\right)=94.69$. An example of searching for the optimal braking mode at this section can be found in Table 1. Search for the optimal solution at the section $S_{f 2} S_{1}^{*}$ was performed using the method of "golden section" [18]. 
According to Table 1, the maximum time intervals $\delta t_{1}\left(\boldsymbol{v}_{2}\right)=\delta t_{2}\left(\boldsymbol{v}_{2}\right)=5.45 \mathrm{~s}$ between cuts can be achieved when $v_{\mathrm{MRP}, 2}=4.69 \mathrm{~m} / \mathrm{s}$ and $v_{\mathrm{GRP}, 2}=5.33 \mathrm{~m} / \mathrm{s}$.

Similarly, at the section $F_{2}^{*} S_{2}^{*}$, we found the optimal braking mode in the group with separating points 5 and 3 , which is at the point $O_{4}$ (see Fig. 8) where $v_{\mathrm{MRP}, 2}=6.90$ $\mathrm{m} / \mathrm{s}, v_{\mathrm{GRP}, 2}=3.54 \mathrm{~m} / \mathrm{s}$, and $\delta t_{1}\left(\boldsymbol{v}_{2}\right)=\delta t_{2}\left(\boldsymbol{v}_{2}\right)=6.87 \mathrm{~s}$.

\section{Conclusions}

The investigations performed make it possible to draw the following conclusions:

1. The braking mode of the middle cut in the design group of three cuts ensuring maximum of the shortest of the intervals on the separating elements in the first and second pairs is always at the boundary of permissible braking mode domain.

2. Specialization of retarder positions of the sloping part of the hump is inappropriate to perform the interval and target regulation of the rolling speed of cuts. Rational distribution of the value of extinguished energy of cut between the retarder positions depends on the location of the separating points along the route of rolling and the base length of the controlled cut. To provide maximal intervals on the separating elements, the retarder positions MRP and GRP should be consistently involved in providing both the necessary separating intervals and the permissible speed of the cut's entry to the park retarder position.

3. As a result of the research, a methodology of searching the braking mode of the middle cut of a design group of three cuts was developed. The methodology makes it possible to move from solving the problem of search for the maximum of nonlinear, non-smooth function of two variables to the solution of the equation with one variable, which significantly accelerates and simplifies the solution of the problem.

Open Access This article is distributed under the terms of the Creative Commons Attribution 4.0 International License (http:// creativecommons.org/licenses/by/4.0/), which permits unrestricted use, distribution, and reproduction in any medium, provided you give appropriate credit to the original author(s) and the source, provide a link to the Creative Commons license, and indicate if changes were made.

\section{References}

1. Zarecky S, Grun J, Zilka J (2008) The newest trends in marshalling yards automation. Transp Probl 3(4):87-95

2. Judge T (2007) Yard management gets smarter. Railw Age $5: 33-34$
3. Ivanchenko VN (2013) Sovremennyie informatsionnyie tehnologii upravleniya slozhnyimi protsessami rasformirovaniya-formirovaniya poezdov (The modern information technology of management of complex processes of breaking-up of trains). Nauka i Transp 2(6):64-69

4. Zhang C, Wei Y, Xiao G et al (2000) Analysis of hump automation in China. Traffic Transp Stud 2000:285-290. https:// doi.org/10.1061/40503(277)45

5. Vasin NN, Kurinskiy VU (2005) Obrabotka videosignalov dlya izmereniya skorosti dvizheniya zheleznodorozhnyih vagonov na sortirovochnoy gorke (Processing video signals for cars speed measuring at the hump). Kompyuternaya Opt 25:185-188

6. Kovalev SM, Liashchenko AM (2013) Nechetko-produktsionnaya model otsenki hodovyih svoystv ottsepov na osnove pertseptivnogo analiza vremennyih ryadov (Fuzzy-production model of the cuts rolling properties evaluation on the basis of perceptual analysis of time series). Aktualnyie voprosyi sovremennoy nauki 30(2): $17-26$

7. Zhukovytskyi IV (2012) Tsifrovaya sistema upravleniya tormozheniem ottsepa zamedlitelyami tormoznoy pozitsii $\mathrm{s}$ diskretnyim izmeritelem skorosti (The digital control system of cuts braking by retarders of retardation position with discrete velocity measurer). Informatsionno-upravlyayuschie sistemyi na zheleznodorozhnom transporte 5:62-66

8. Nazarov OA (2016) Reduction in cuts speed at the beginning of a sorting sidings, equipped with quasi-continuous speed control system. Sci Transp Prog 4(64):47-54. https://doi.org/10.15802/ stp2016/77881

9. Bessonenko SA, Ivanchenko VN, Liashchenko AM (2013) Matematicheskaya model rascheta parametrov intervalnogo tormozheniya ottsepov i peremennyih skorostey rospuska sostavov (Mathematical model of parameters determination of cuts interval braking and variable speed of train sorting). Vestnik RGUPS 1(49):55-65

10. Yao J, Li X, Liu H (2001) Study on neural network based spaceinterval speed-control model. China Railw Sci 2:127-133

11. Puchkov EV (2010) Primenenie neyroemulyatora NeuroNADS dlya opredeleniya stupeni i vremeni tormozheniya pri upravlenii gorochnyimi zamedlitelyami (Application of neyroemulyator NeuroNADS for determination of degree and time of breaking in hump retarders controlling). Inzhenernyiy vestnik Dona 4(14):157-162

12. Bobrovskyi V, Kozachenko D, Dorosh A et al (2016) Probabilistic approach for the determination of cuts permissible braking modes on the sorting humps. Transp Probl 11(1):147-155. https://doi.org/10.20858/tp.2016.11.1.14

13. Bobrovskyi VI, Rogov NV (2004) Optimizatsiya rezhimov regulirovaniya skorosti ottsepov pri rospuske sostavov na gorkah (Optimization of cuts speed control modes during train brakingup at the humps). Sci Transp Prog 4:174-182

14. Zhang C, Li Y (2011) Research on multi-objective optimization of vertical section of the rolling down zone on hump. In: 2011 Seventh international conference on natural computation, pp 1270-1274. https://doi.org/10.1109/ICNC.2011.6022353

15. Avramovic $Z$ (1995) Method for evaluating the strength of retarding steps on a marshalling yard hump. Eur J Oper Res 85(3):504-514. https://doi.org/10.1016/0377-2217(92)00129-9

16. Bobrovskyi VI, Kozachenko DN (2010) Modelirovanie protsessa skatyivaniya ottsepov s sortirovochnoy gorki (Cuts rolling-down from the hump process modelling). Zb. nauk. prats DETUT: Seriya "Transportni sistemi i tehnologyi" 16:20-29

17. Wald A (1945) Statistical decision functions which minimize the maximum risk. Ann Math 46(2):265-280

18. Press W, Teukolsky S, Vetterling W et al (2007) Numerical recipes. The art of scientific computing. Cambridge University Press, New York 